\title{
REGISTROS DE MURCIÉLAGOS PARA EL ESTADO DE GUERRERO, MÉXICO
}

\author{
José Alberto Almazán-Catalán1, Alejandro TABoAdA-SAlgado², \\ Cornelio SÁNCHEZ-HERNÁNDEZ ${ }^{1}$, María de Lourdes ROMERO-ALMARAZ ${ }^{3}$, \\ Yanet Q. JiMÉNEZ-SALMERóN ${ }^{1}$ y Ezequiel GUERRERo IBARRA ${ }^{1}$
}

1Departamento de Zoología, Instituto de Biología, Universidad Nacional Autónoma de México, A.P.

70-153, Coyoacán, C.P. 04510, MÉXICO, D.F. almazancatalan@hotmail.com, cornelio@servidor.unam.mx, quintina76@hotmail.com, ezequielguerrero_50@hotmail.com

2Recursos Genéticos y Productividad-Ganadería, Colegio de Postgraduados, Montecillo 56230,

Texcoco, Estado de México, MÉXICO taboada-salgado@hotmail.com

${ }^{3}$ Escuinapa No. 92 bis. Col. Pedregal de Santo Domingo, C.P. 04360, México, D.F., MÉXICO 1romero22@hotmail.com

Almazán-Catalán, J. A., A. Taboada-Salgado, C. Sánchez-Hernández, Ma. de L. Romero-Almaraz, Y. Q. Jiménez-Salmerón y E. Guerrero Ibarra. 2009. Registros de murciélagos para el estado de Guerrero, México. Acta Zool. Mex. (n. s.) 25(1): 177-185.

RESUMEN. Se aporta información sobre la distribución, hábitat, medidas y condición reproductiva de nueve especies de murciélagos del estado de Guerrero, México. Cuatro especies representan el segundo registro para el estado (Choeroniscus godmani, Myotis auriculus apache, Myotis thysanodes aztecus y Nyctinomops femorosaccus), tres representan el tercer registro (Musonycteris harrisoni, Chiroderma salvini scopaeum y Enchisthenes hartii), y dos son el cuarto registro (Hylonycteris underwoodi minor y Myotis californicus mexicanus).

Palabras clave: Guerrero, murciélagos, distribución, reproducción.

Almazán-Catalán, J. A., A. Taboada-Salgado, C. Sánchez-Hernández, Ma. de L. Romero-Almaraz, Y. Q. Jiménez-Salmerón \& E. Guerrero Ibarra. 2009. Records of bats from the state of Guerrero, México. Acta Zool. Mex. (n. s.) 25(1): 177-185.

ABSTRACT. Information on geographic distribution, habitat, measurements, and reproductive condition of nine species of bats from Guerrero, Mexico, is provided. Four species are the second records for the State (Choeroniscus godmani, Myotis auriculus apache, Myotis thysanodes aztecus and Nyctinomops femorosaccus), three are the third record (Musonycteris harrisoni, Chiroderma salvini scopaeum and Enchisthenes hartii), and two are the fourth record (Hylonycteris underwoodi minor and Myotis californicus mexicanus).

Key words: Guerrero, bats, distribution, reproduction.

\section{INTRODUCCIÓN}

Las características fisiográficas, climáticas y la gran diversidad ecológica que presentan las provincias bióticas del estado de Guerrero (Cuenca del Balsas, Sierra 
Madre del Sur, Planicie Costera del Pacífico y Sierra de Taxco), han favorecido la presencia de una gran riqueza de especies de mamíferos (Almazán-Catalán et al. 2005). Por otra parte, lo accidentado de la topografía, la falta de vías de comunicación y los problemas políticos y sociales que imperan en el estado han limitado el trabajo de investigación y el desarrollo de las comunidades en gran parte de su territorio y principalmente en la Sierra Madre del Sur, lo anterior ha propiciado que a pesar de la gran riqueza de mamíferos de Guerrero, éstos hayan sido pobremente estudiados, como puede confirmarse con la descripción reciente de dos especies nuevas de roedores (Bradley et al. 2004, Romo-Vázquez et al. 2005) y la ampliación en la distribución de dos especies más de ratones y una de murciélagos (Cervantes et al. 2004, Almazán-Catalán et al. 2005). Con este trabajo se contribuye al conocimiento de la distribución de los murciélagos, con el registro de nueve especies que se citan por segunda, tercera o cuarta ocasión para el estado, a la vez que se aporta información sobre su historia natural y actividad reproductiva.

\section{MATERIAL Y MÉTODOS}

Área de estudio. Las localidades de captura que se encuentran en los municipios Tlalchapa y Eduardo Neri, corresponden a la Cuenca del Balsas, que pertenece a la unidad mesoclimática (UMC) muy cálida semiseca, la cual presenta temperatura media anual superior a $26^{\circ} \mathrm{C}$ y precipitaciones entre 600 y $900 \mathrm{~mm}$; la altitud varía del nivel del mar hasta los $600 \mathrm{~m}$, y la vegetación dominante es bosque tropical caducifolio (Meza \& López-García 1997). Las localidades de los municipios Leonardo Bravo y Chilpancingo de los Bravo se ubican en la Sierra Madre del Sur, el primero pertenece a la UMC templada húmeda, con temperaturas medias anuales entre 15 y $18^{\circ} \mathrm{C}$, y precipitación anual de $1,100 \mathrm{a}$ $1,500 \mathrm{~mm}$; la altitud varía de 2,000 a $2,800 \mathrm{~m}$ snm y la vegetación pertenece a bosque de pino, bosque de encino y bosque mesófilo de montaña. Por su parte, las localidades de Chilpancingo de los Bravo se encuentran en la UMC cálida húmeda, con una temperatura media anual de 22 a $26^{\circ} \mathrm{C}$, precipitación anual entre 1,100 y $1,500 \mathrm{~mm}$; y altitudes de 600 a $1,300 \mathrm{~m}$ snm; la vegetación corresponde a bosque de encino, bosque tropical caducifolio y bosque de galería (Meza \& López-García 1997).

Trabajo de campo. Los murciélagos se capturaron entre abril de 2003 y noviembre de 2007, con redes de niebla de varios tamaños, colocadas entre la vegetación o sobre cuerpos de agua. Los especímenes se encuentran preservados en piel, cráneo y esqueleto o en alcohol al 70\%. Las medidas se citan en el cuadro 1 en milímetros y el peso en gramos. 


\section{RESULTADOS}

Choeroniscus godmani (Thomas, 1903). Se examinaron dos hembras adultas inactivas sexualmente, una se capturó el 25 de abril del 2003, en el municipio de Leonardo Bravo, Los

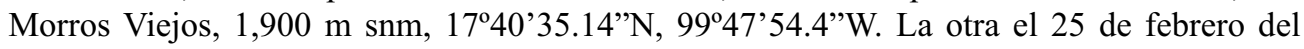
2006, en el municipio de Leonardo Bravo, Los Cajones, $2 \mathrm{~km} \mathrm{~S}$ Carrizal de Bravo, 2,560 m snm, $17^{\circ} 36^{\prime} 13^{\prime \prime} \mathrm{N}, 9^{\circ} 50^{\prime} 09^{\prime \prime} \mathrm{W}$. Los especímenes se caracterizan por tener el rostro más largo y estrecho que las especies del género Glossophaga, las orejas son cortas y redondas; la coloración es morena negruzca algo sucia, debido a la coloración del pelo que tiene tres bandas, la basal de color gris obscuro, la intermedia gris pálido y la punta morena negruzca. La región ventral de la garganta y cuello es color moreno negruzco y el vientre gris pálido. Los especímenes están preservados en piel, cráneo y partes de esqueleto, en buenas condiciones. Se capturaron en redes colocadas entre la vegetación de pino y encino en la localidad de Los Morros Viejos y en bosque mesófilo de montaña en Los Cajones. Su captura representa el segundo y tercer registro para el estado y aumenta en aproximadamente $80 \mathrm{~km}$ al sureste de su localidad previamente citada para Guerrero, de $1.5 \mathrm{~km}$ SE de San Andrés [Juan] de la Cruz, $700 \mathrm{~m}$ snm (Handley 1966). Las medidas del espécimen examinado son similares a las citadas por Gardner (1962) y Goodwin (1969), aunque en nuestro espécimen la HMD es mayor (7.96 Vs 7.4 o menos).

Hylonycteris underwoodi minor Phillips and Jones, 1971. Se examinó un macho adulto capturado en abril del 2003, en el municipio de Leonardo Bravo, $700 \mathrm{~m}$ al W de Los Morros, $2,000 \mathrm{~m} \mathrm{snm}, 17^{\circ} 41^{\prime} 11^{\prime \prime} \mathrm{N}, 99^{\circ} 47^{\prime} 18^{\prime \prime} \mathrm{W}$. El espécimen se caracteriza por tener el rostro más fino y menos respingado, así como las orejas más cortas y redondas que Choeroniscus godmani; la coloración es morena negruzca, y el pelo tiene tres o cuatro bandas, la basal es gris obscura, la intermedia y de mayor extensión, gris pálida y la punta negruzca; algunos pelos pueden tener una cuarta banda en donde las puntas son grises, lo que les da un aspecto ligeramente canoso; la parte ventral es de color más pálido que la dorsal. El espécimen está preservado en piel y cráneo en buenas condiciones, fue capturado en bosque de pino-encino. Su captura representa el cuarto registro para Guerrero y aumenta su distribución en $19 \mathrm{~km}$ al noroeste de su localidad más cercana en el municipio de Chilpancingo de los Bravo, Omiltemi, Laguna de Agua Fría (León-Paniagua \& Romo-Vázquez 1991).

Musonycteris harrisoni Schaldach y McLaughlin, 1960. Se examinó una hembra adulta inactiva sexualmente, capturada en julio del 2004 en el municipio Eduardo Neri, en la barranca Xochipala, a $2 \mathrm{~km} \mathrm{SW}$ de Mezcala, $600 \mathrm{~m}$ snm, 1754'40.54”N, 99³6’41.14”W. La piel está preservada en condiciones regulares, y se conserva el cráneo y partes de esqueleto. Se capturó en una red colocada en vegetación de bosque tropical caducifolio con dominancia de árboles del género Bursera y cactáceas como Neobuxbaumia mezcalaensis y Pachycereus weberi. La longitud del rostro hasta la base de los nasales se caracteriza por ser mayor que la mitad de la longitud del cráneo. Su captura representa el tercer registro y el más norteño para Guerrero, porque había sido citado anteriormente para el Cañón del Zopilote, a $23.3 \mathrm{~km}$ (por carretera) $\mathrm{N}$ de Zumpango, $610 \mathrm{~m}$ snm (Winkelmann 1962) y de $2 \mathrm{~km}$ SE de Tecpan de Galeana, $120 \mathrm{~m}$ snm (Ramírez-Pulido et al. 1977). Por otra parte, la localidad citada es intermedia entre la del estado de Morelos, 2 y $3 \mathrm{~km}$ NW de Palpan (Álvarez-Castañeda \& López-Forment 1995) y la de Guerrero, 2 km SE de Tecpan, 120 m snm (Ramírez-Pulido et 
al. 1977). Tanto las medidas del cuerpo como las del cráneo corresponden con las citadas por otros autores como Schaldach \& McLaughlin (1960) y Téllez \& Ortega (1999).

Chiroderma salvini scopaeum Handley, 1966. Se examinaron dos hembras adultas inactivas, capturadas en el municipio Chilpancingo de los Bravo, una en octubre del 2006 en el río Azinyahualco, a $4 \mathrm{~km} \mathrm{NW}$ de Zoyatepec, $1,100 \mathrm{~m} \mathrm{snm}, 17^{\circ} 22^{\prime} 08^{\prime \prime} \mathrm{N}, 99^{\circ} 33^{\prime} 45^{\prime \prime} \mathrm{W}$, y la otra en noviembre del 2007 en el deportivo Mitzumaro, a $2 \mathrm{~km}$ SE de Chilpancingo, 1,300 m snm, $17^{\circ} 32^{\prime} 00^{\prime} \mathrm{N}, 9^{\circ} 28^{\prime} 57^{\prime}$ 'W. Los especímenes se caracterizan por tener el dorso de color moreno; con el pelo tricolor, de base grisácea, intermedia pálida y punta morena; tienen una línea blanca media dorsal estrecha, y las líneas del rostro están bien marcadas; la longitud del antebrazo es mayor de 41 y menor de $53 \mathrm{~mm}$ y los incisivos superiores internos son convergentes. El primer espécimen se capturó en una red que se colocó en bosque de galería rodeado de bosque tropical caducifolio y cultivos de maíz; se encuentra preservado en piel y esqueleto; el segundo, en una red colocada a la entrada de una barranca con bosque tropical caducifolio, a las 21:15 h; y se encuentra preservado en piel. La captura de estos especímenes representan el tercer y cuarto registro para el estado, citado previamente para Acahuizotla por Handley (1965) y Carter et al. (1966), y para el municipio de Taxco de Alarcón en el Parque Estatal El Huizteco (León \& Romo 1991, León-Paniagua \& Romo-Vázquez 1993). Las medidas de nuestros especímenes concuerdan con las citadas por Handley (1965).

Enchisthenes hartii (Thomas, 1892). Se examinaron dos hembras adultas lactando, que fueron capturadas en agosto del 2006, en el municipio de Chilpancingo de los Bravo, una a

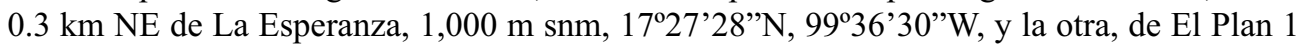

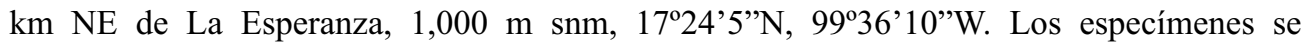
caracterizan por tener el rostro color moreno chocolate, con las líneas supraorbitales ligeramente pálidas; con pelo abundante en el antebrazo y el borde del uropatagio; los terceros molares superior e inferior están bien desarrollados, y afectan la forma lateral de los dentarios; los incisivos inferiores internos son unicúspides. Los especímenes se encuentran preservados en piel, cráneo y partes de esqueleto; fueron capturados en redes colocadas sobre un arroyo en bosque de galería y entre árboles de guayaba (Psidium guajava) y plátano (Musa sp.), a las 22:00 h. Sus capturas representan el tercero y cuarto registro para el estado, citado anteriormente para el municipio de Tecpan de Galeana, $28 \mathrm{~km}$ (por carretera) $\mathrm{N}$ de Tecpan, 350 m snm (Ramírez-Pulido \& López-Forment 1979); y para el municipio de Atoyac, Retrocesos, 1,550 m snm (León-Paniagua \& Romo-Vázquez 1991, Arroyo-Cabrales \& Owen 1996). Las medidas corresponden con las citadas por Arroyo-Cabrales \& Owen (1996).

Myotis auriculus apache Hoffmeisteir y Krutzch, 1955. Se examinó un macho adulto con testículos en posición inguinal de $4 \times 3 \mathrm{~mm}$, capturado en abril del 2004, en el municipio

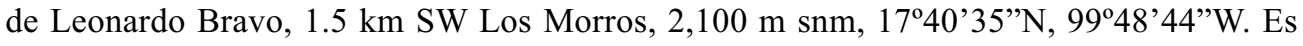
de color moreno, con pelo suave y escaso, con la base gris negruzca y las puntas moreno gamuza o moreno canela. No tiene máscara negra, las orejas son largas y de color negro, pero las membranas de las alas y el uropatagio son más pálidas (Miller \& Allen 1928). El espécimen está preservado en piel y cráneo, este último roto en la parte occipital derecha. $\mathrm{Su}$ captura representa el segundo registro para el estado y aumenta aproximadamente en $100 \mathrm{~km}$ al sur de su localidad anterior en el municipio Ixcateopan de Cuauhtémoc, $10 \mathrm{~km}$ NW de Ixcateopan (León-Paniagua \& Romo-Vázquez 1991, León-Paniagua \& Romo- 
Vázquez 1993). Las medidas del espécimen corresponden parcialmente con las citadas por Miller \& Allen (1928).

Myotis californicus mexicanus (de Saussure, 1860). Se capturó un macho adulto en abril del 2006, en el municipio de Leonardo Bravo, Las Truchas, 3 km SE de Carrizal de Bravo, $2,400 \mathrm{~m} \mathrm{snm}, 17^{\circ} 35^{\prime} 54^{\prime \prime} \mathrm{N}, 9^{\circ} 49^{\prime} 39^{\prime \prime} \mathrm{W}$. Se encuentra preservado en piel, cráneo y partes de esqueleto en buenas condiciones. Se caracteriza porque el rostro y las orejas son negros e integran una máscara, la coloración del dorso es moreno canela; el pelo es bicolor con la base negra en las $2 / 3$ partes y la punta moreno canela quemada. En el vientre el color es pálido, con el pelo bicolor, con la base negra en 3/4 partes y las puntas gamuza pálida. El rostro es largo, estrecho y se levanta abruptamente hacia la caja craneal, en un ángulo de $140^{\circ}$; los premolares se encuentran en línea; el calcáneo está quillado y las patas son pequeñas; lo que concuerda con algunas de las características citadas por Miller \& Allen (1928). Además de que no tiene pelo en la parte dorsal del antebrazo, ni en la parte distal del brazo, y por la parte ventral el pelo es escaso, lo que lo diferencia de M. volans (Yancey 1997). A pesar de que el espécimen tenía los testículos inguinales, en las orejas había unas glándulas dorsales muy desarrolladas $(5.6 \times 3.1 \mathrm{~mm})$, lo que indica que iniciaba su madurez reproductiva. La captura de este espécimen representa el cuarto registro para Guerrero y se encuentra a $20 \mathrm{~km}$ al norte a partir de su localidad más próxima, en el municipio de Chilpancingo de los Bravo, Omiltemi (León-Paniagua \& Romo-Vázquez 1991, Jiménez-Almaraz et al. 1993).

Myotis thysanodes aztecus Miller y G. M. Allen, 1928. Se examinaron cinco hembras adultas capturadas en abril del 2004, en el municipio de Leonardo Bravo; una de $0.7 \mathrm{~km}$ al

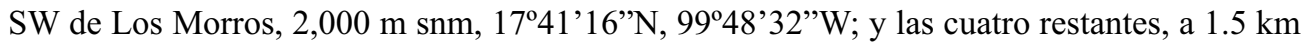

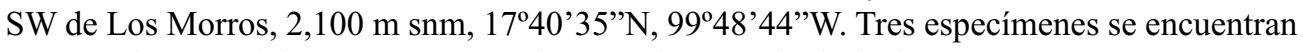
preservados en piel, cráneo y esqueleto, y dos en alcohol al 70\%. Los especímenes se caracterizan por tener un fleco de pelo en el borde del uropatagio; fueron capturados en redes colocadas sobre un arroyo, entre las 19:00 y las 21:00 h, en bosque de pino-encino. Su captura representa el segundo registro para Guerrero y amplía su distribución en $45 \mathrm{~km}$ aproximadamente hacia el noreste de la localidad citada anteriormente del municipio General Heliodoro Castillo, 1 km E de Puerto del Gallo (León-Paniagua \& Romo-Vázquez 1991). Cuatro hembras estaban preñadas con un embrión cada una $(14 \times 8,14 \times 10,15 \times 12,16 \times 11)$ y la otra fue inactiva sexualmente. El tamaño de los embriones compagina con el período de reproducción citado para la especie (O'Farrel \& Studier 1980), por lo que creemos que es residente en estas áreas del estado. Las medidas somáticas y craneales son similares a las citadas por Miller \& Allen (1928) y O'Farrell \& Studier (1980).

Nyctinomops femorosaccus (Merriam, 1889). Se examinó un macho adulto con testículos inguinales de $4 \times 2 \mathrm{~mm}$, que se capturó en diciembre del 2005, en el municipio de Tlalchapa, en La Poza de Lico, a $1 \mathrm{~km}$ al W de Cuahulotitlán, $380 \mathrm{~m}$ snm, 18²0'22'N, $100^{\circ} 20^{\prime} 19^{\prime \prime} \mathrm{W}$. Se caracteriza por tener el primer premolar superior de forma cuadrada y porque el borde posterior del tercer molar termina antes que el palatino, características que lo diferencian de $N$. laticaudatus y de $N$. aurispinosus, donde el premolar es de forma aproximada de trapecio y el borde posterior del tercer molar y del palatino terminan al mismo nivel (Hall 1981). El espécimen se encuentra preservado en piel, cráneo y partes de esqueleto; fue capturado en una red colocada sobre una poza de agua poco profunda, a las 20:30 h, 
Almazán-Catalán et al. Murciélagos de Guerrero, México

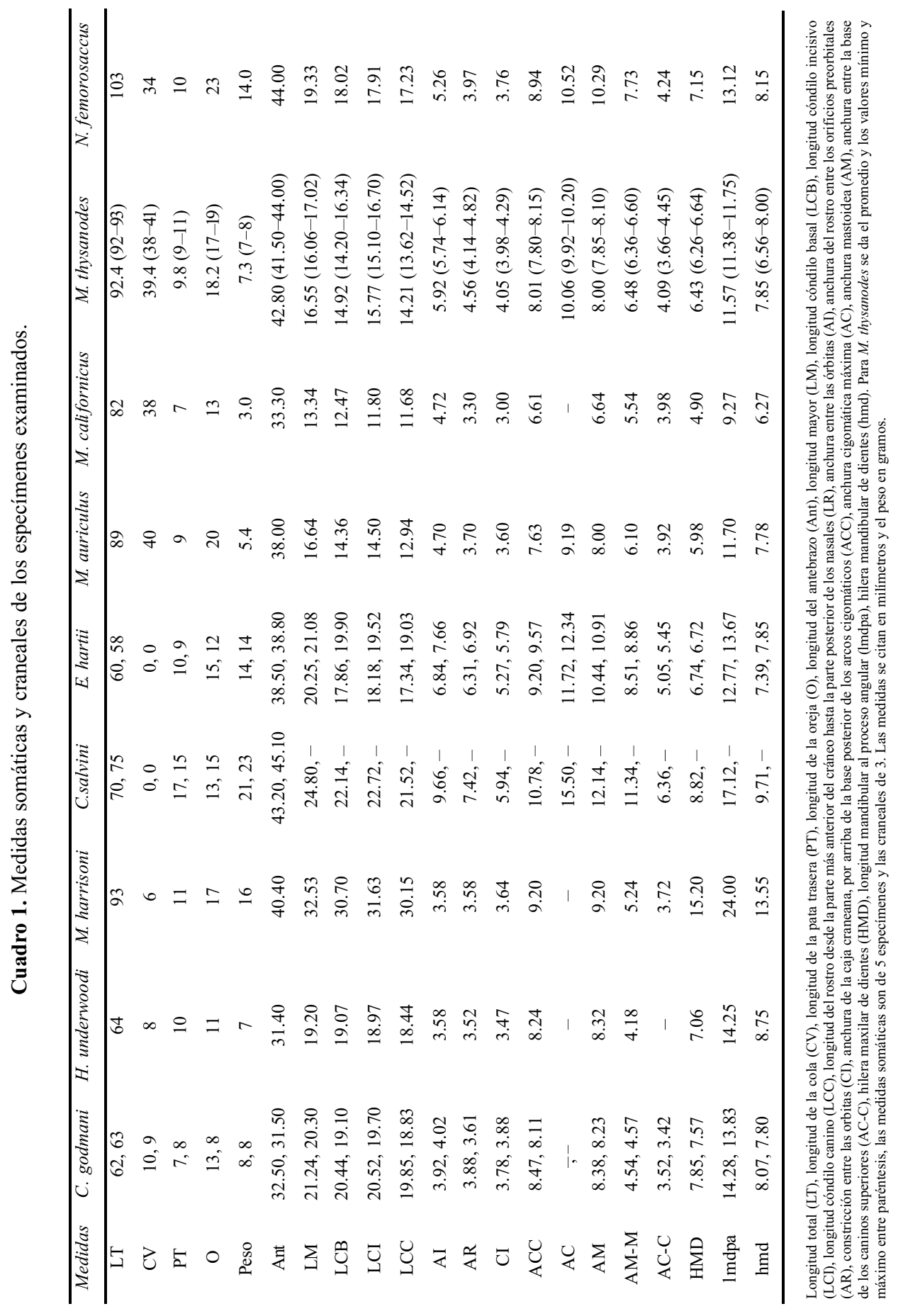


localizada entre la vegetación de bosque tropical caducifolio, con predominancia de árboles de guamúchil (Pithecellobium dulce), rodeado por cultivos de maíz y calabaza. La captura de este espécimen aumenta en $96 \mathrm{~km}$ al oeste de su localidad anterior localizada en el municipio de Huitzuco de los Figueroa, Poza de Los Santos, $1.5 \mathrm{~km} \mathrm{~N}$ de Tlaxmalac, $940 \mathrm{~m} \mathrm{snm}$ (Almazán-Catalán et al. 2005) y representa el segundo registro para el estado.

\section{DISCUSIÓN}

Cuatro especies representan el segundo registro (C. godmani, M. auriculus, $M$. thysanodes y N. femorosaccus), tres el tercer registro (M. harrisoni, C. salvini y E. hartii), y dos el cuarto registro (H. underwoodi y M. californicus) para Guerrero. Lo anterior es interesante, si se considera que las nueve especies representan $6.5 \%$ de los murciélagos de Guerrero (Ramírez-Pulido \& Castro-Campillo 1990, 1994; RamírezPulido et al. 1986, 2000; Almazán-Catalán et al. 2005), lo que enfatiza la falta de trabajos mastozoológicos en el estado. De los especímenes capturados, C. godmani se había citado para Guerrero, hace más de 40 años (Handley 1966); M. harrisoni, más de 30 (Ramírez-Pulido et al. 1977), E. hartii, M. auriculus y M. thysanodes desde 1991 (León-Paniagua \& Romo-Vázquez 1991) y C. salvini desde 1993 (LeónPaniagua \& Romo-Vázquez 1993). Por otra parte, M. harrisoni se considera una especie en peligro y E. hartii como protegida, de acuerdo con la NOM-059-ECOL2001 (Secretaría de Medio Ambiente y Recursos Naturales, 2002). Es importante señalar que de C. godmani, H. underwoodii, E. hartii y M. thysanodes, sólo se había registrado la localidad de captura (Handley 1966, León-Paniagua \& Romo-Vázquez 1991, Arroyo-Cabrales \& Owen 1996). Por lo que este estudio amplía la información sobre su distribución y señala por primera ocasión aspectos de su hábitat, reproducción y medidas.

AGRADECIMIENTOS. Deseamos hacer patente nuestro reconocimiento a las autoridades de SEMARNAT por el permiso de colector otorgado a Dr. C. Sánchez Hernández, números SGPA/DGVS/05437 (2004), SGPA/DGVS/10415 (2005) y SGPA/DGVS/06541 (2006). A la M. en C. E. Beltrán Sánchez por la donación de una piel de Chiroderma salvini y al Ecólogo L. Sánchez Vázquez por su ayuda en el trabajo de campo.

\section{LITERATURA CITADA}

Almazán-Catalán, J.A., C. Sánchez-Hernández \& M.L. Romero Almaraz. 2005. Registros sobresalientes de mamíferos del estado de Guerrero, México. Acta Zoológica Mexicana (n.s.). 21:155-157.

Álvarez-Castañeda, S.T. \& W. López-Forment, C. 1995. Datos sobre los mamíferos del área aledaña a Palpan, Morelos, México. Anales del Instituto de Biología, Universidad Nacional Autónoma de México. 66:123-133.

Arroyo-Cabrales, J. \& R.D. Owen. 1996. Intraspecific variation and phenetic affinities of Dermanura hartii, with reapplication of the specific name Enchisthenes hartii. Pp. 67-81. In: H.H. Genoways and R.J. Baker (Eds.). Contributions in Mammalogy: A memorial volume honoring Dr. J. Knox Jones, Jr. Texas Tech University Press, Lubbock, Texas. 
Bradley, R.D., F. Mendez-Harclerode, M.J. Hamilton \& G. Ceballos. 2004. A new species of Reithrodontomys from Guerrero, Mexico. Occasional Papers, Texas Tech University. 231:1-12.

Carter, D.C., R.H. Pine \& W.B. Davis. 1966. Notes on middle american bats. Southwestern Naturalist. 11:488-499.

Cervantes, F.A., J.N. Ramírez-Vite, S. Ramírez-Vite \& C. Ballesteros. 2004. New records of mammals from Hidalgo and Guerrero, México. Southwestern Naturalist. 49:122-124.

Gardner, A.L. 1962. Bat records from the Mexican states of Colima and Nayarit. Journal of Mammalogy. 43:102-103.

Goodwin, G.G. 1969. Mammals from the state of Oaxaca, Mexico, in the American Museum of Natural History. Bulletin of the American Museum of Natural History. 141:1-269.

Hall, E.R. 1981. The mammals of North America. John Wiley and Sons, New York.

Handley, C.O., Jr. 1965. Descriptions of new bats (Chiroderma and Artibeus) from Mexico. Anales del Instituto de Biología, Universidad Nacional Autónoma de México, Serie Zoología. 36:297-301.

Handley, C.O., Jr. 1966. Descripttions of new bats (Choeroniscus and Rhinophylla) from Colombia. Proceedings of the Biological Society of Washington. 79:83-88.

Jiménez-Almaraz, J. Juárez-Gómez \& L. León-Paniagua. 1993. Mamíferos. Pp. 505-549. In: I. Luna V. \& J. Llorente B. (Eds.). Historia Natural del Parque Ecológico Estatal Omiltemi, Chilpancingo, Guerrero. Comisión Nacional para el Conocimiento y Uso de la Biodiversidad, Universidad Nacional Autónoma de México, México, D. F.

León-Paniagua, L. \& E. Romo-Vázquez. 1991. Catálogo de la colección de mamíferos del Museo de Zoología de la Facultad de Ciencias, Universidad Nacional Autónoma México. Serie Catálogos del Museo de Zoología "Alfonso L. Herrera". Facultad de Ciencias, Universidad Nacional Autónoma de México, México, D. F.

León Paniagua, L. \& E. Romo Vázquez. 1993. Mastofauna de la sierra de Taxco, Guerrero. Pp. 45 64. In: R.A. Medellín \& G. Ceballos (Eds.). Avances en el Estudio de los Mamíferos de México. Publicaciones Especiales, Volumen 1, Asociación Mexicana de Mastozoología, A. C., México, D. F.

Meza, L. \& J. López-García. 1997. Vegetación y mesoclimas de Guerrero. Pp. 1-51. In: N. Diego \& R.M. Fonseca (Eds.). Estudios Florísticos en Guerrero. Facultad de Ciencias, Universidad Nacional Autónoma de México, México, D. F.

Miller, G.S., Jr. \& G.M. Allen. 1928. The American bats of the genera Myotis and Pizonyx. Bulletin of the United States National Museum. 144:1-218.

O'Farrell, M.J. \& E.H. Studier. 1980. Myotis thysanodes. Mammalian Species. 137:1-5.

Ramírez-Pulido, J., A. Martínez \& G. Urbano. 1977. Mamíferos de la Costa Grande de Guerrero, México. Anales del Instituto de Biología, Universidad Nacional Autónoma de México, Serie Zoología. 48:243-292.

Ramírez-Pulido, J. \& W. López-Forment. 1979. Additional records of some Mexican bats. Southwestern Naturalist. 24:541-544.

Ramírez Pulido, J., M.C. Britton, A. Perdomo \& A. Castro. 1986. Guía de los mamíferos de México referencias hasta 1983. Universidad Autónoma Metropolitana, Unidad Iztapalapa, México, D. F.

Ramírez-Pulido J. \& A. Castro-Campillo. 1990. Bibliografía reciente de los mamíferos de México 1983-1988. Universidad Autónoma Metropolitana, Unidad Iztapalapa, México, D. F.

Ramírez-Pulido J. \& A. Castro-Campillo. 1994. Bibliografía reciente de los mamíferos de México 1989-1993. Universidad Autónoma Metropolitana, Unidad Iztapalapa, México, D. F.

Ramírez-Pulido J., A. Castro-Campillo, M. A. Armella \& A. Salome-Méndez. 2000. Bibliografía reciente de los mamíferos de México 1994-2000. Universidad Autónoma Metropolitana, Unidad Iztapalapa, México, D. F. 
Romo-Vázquez, E., L. León \& O. Sánchez. 2005. A new species of Habromys (Rodentia: Sigmodontinae) from Mexico. Proceedings of the Biological Society of Washington. 118:605-611.

Schaldach, W.J. \& C.A. McLaughlin. 1960. A new genus and species of Glossophagine bat from Colima, México. Contributions in Science, Los Angeles County Museum. 37:1-8.

Secretaría de Medio Ambiente y Recursos Naturales. 2002. Norma Oficial Mexicana NOM-059ECOL-2001, protección ambiental-especies nativas de México de flora y fauna silvestres-categorías de riesgo y especificaciones para su inclusión, exclusión o cambio-lista de especies en riesgo. Diario Oficial, 6 de Marzo del 2002:1-56.

Téllez, G. \& J. Ortega. 1999. Musonycteris harrisoni. Mammalian Species. 622:1-3.

Winkelmann, J.R. 1962. Mammal records from Guerrero and Michoacan, Mexico. Journal of Mammalogy. 43:108-109.

Yancey, F.D.II. 1997. The Mammals of Big Bend Ranch State Park, Texas. Special Publications of The Museum, Texas Tech University Press Press, Lubbock, Texas.

Recepción: 14 de septiembre 2007

Aceptación: 26 de noviembre 2008 